\title{
Focal segmental glomerulosclerosis as the leading cause of idiopathic nephrotic syndrome: a different spectrum revealed by mandatory renal biopsy in all patients
}

\author{
*Swarnali Mani Joardar ${ }^{1}$, Rita Chatterjee ${ }^{2}$, Shubhadeep Das ${ }^{2}$, Siddhartha Mani ${ }^{2}$
}

Sri Lanka Journal of Child Health, 2016; 45(2):76-83

\begin{abstract}
Introduction: Idiopathic nephrotic syndrome (INS) is associated with varied glomerular lesions. The evidence so far confirms a higher incidence of minimal change disease (MCD) than other subtypes in children. Different newer trials and review literature suggest a 2-fold increase in the incidence of focal segmental glomerulosclerosis (FSGS) in recent decades.
\end{abstract}

Objective: To determine the histopathology of INS following mandatory renal biopsy

Method: The patients attending the Department of Paediatric Medicine with a diagnosis of INS were selected for the study. A confirmatory diagnosis was established. Thereafter all patients underwent a mandatory renal biopsy (after exclusion of any absolute contraindication) and histopathology examination. Different findings were compared between MCD and Non-MCD groups (those having histological lesion other than MCD).

Results: There were 52 children suffering from nephrotic syndrome (NS) whom we included in our study. The most frequently noted glomerular lesion was FSGS in $45 \%$. MCD was noted in $27 \%$, mesangioproliferative glomerulonephritis (MSGN) in $12 \%$, membranoproliferative glomerulonephritis (MPGN) in $10 \%$ and membranous glomerulonephritis (MGN) in 6\%. The incidence of relapse was significantly higher in the Non-MCD group $(\mathrm{p}=0.02)$.

Conclusions: In our study, a mandatory renal biopsy in all patients found FSGS to be the most

${ }^{1}$ College of Medicine and Sagore Dutta Hospital, India, ${ }^{2}$ Medical College Kolkata, India

*Correspondence: swarnalijoardar@gmail.com (Received on 15 May 2015: Accepted after revision on 19 June 2015)

The authors declare that there are no conflicts of interest

Personal funding was used for this project.

Open Access Article published under the Creative

Commons Attribution CC-BY CC common glomerular lesion among paediatric age group patients suffering from INS.

(Key words: Focal segmental glomerulosclerosis, idiopathic nephrotic syndrome, renal biopsy)

DOI: http://dx.doi.org/10.4038/sljch.v45i2.7979

\section{Introduction}

Nephrotic syndrome (NS) in children remains a common cause for hospital admissions in paediatric wards with an annual incidence of 2-7 per 100,000 children, and a prevalence of $12-16$ per $100,000^{1}$. Idiopathic nephrotic syndrome (INS), is associated with glomerular diseases intrinsic to the kidney including minimal change disease (MCD), focal segmental glomerulosclerosis (FSGS), membranous glomerulonephritis (MGN), membranoproliferative glomerulonephritis (MPGN), mesangioproliferative glomerulonephritis (MSGN) and others. MCD still remains the most prevalent cause of INS. The frequency of FSGS associated with INS appears to be increasing. A review of the literature suggested a 2 -fold increase in the incidence of FSGS ${ }^{2}$. However, other studies have not found such evidence ${ }^{3}$. In almost all of these studies, renal biopsy was only selectively done in patients according to the recommendations for renal biopsy. Other patients who did not meet the criteria for biopsy were mostly considered as presumed MCD. Thus we decided to conduct a study where renal biopsy will be done in all patients excepting only those with contraindications. This may reflect the actual proportion of histological subtypes among INS patients.

\section{Method}

The patients attending the Department of Paediatric Medicine in Medical College, Kolkata with a provisional diagnosis of INS were selected for this observational cross-sectional study after proper assessment of inclusion and exclusion criteria:

Inclusion criteria:

(i) All diagnosed patients of INS.

(ii) Age group - more than 6 months to less than 12 years. 
Exclusion criteria:

(i) Any chronic disease / comorbidity other than NS.

(ii) Patients with any contraindications for renal biopsy.

A proper and relevant history and clinical examination were performed. Signs and symptoms that may suggest a secondary condition were searched for e.g. malar rash, adenopathy, arthritis, etc. Anthropometric measurements including body weight, height, etc. were taken on admission and weight was recorded daily. After resolution of oedema to the maximum possible extent, the final weight measurement was recorded to assess the nutritional status of the patient. Thus we tried to eliminate the confounding effect of oedema on assessment of nutritional status. Finally they were thoroughly investigated to diagnose INS. Other tests, to exclude secondary nephrotic syndrome, were done as required e.g. ANF, Hepatitis B, Hepatitis C, and HIV serology etc.

Renal biopsy was done in 56 patients having a confirmatory diagnosis of INS, irrespective of whether biopsy was indicated or not, after obtaining proper ethical clearance and after ruling out contraindications to the procedure by doing prothrombin time / INR, urine culture and ultrasonography. Renal biopsy was done under ultrasonic guidance from the back at the renal angle using an appropriate sized BARD biopsy gun. At least 10 glomeruli examined during histopathology was considered adequate. The same experienced pathologist performed histopathology. Four patients were excluded due to inadequacy of the renal biopsy specimen. The final statistical analyses were performed on 52 patients. Standard definitions to define the course of nephrotic syndrome were used ${ }^{4}$.

Statistical analysis: The observed results were compared between different sub-groups using appropriate standard statistical tests. Unpaired Student's t test was used for normally distributed variables, Chi-square test was used for nominal categorical variables. $\mathrm{P}$ value $<0.05$ was taken as significant.

Ethical considerations: All human studies have been reviewed by the ethics committee of our Institution and have therefore been performed in accordance with the ethical standards laid down in an appropriate version of the 2000 Declaration of Helsinki as well as the Declaration of Istanbul 2008. Parents/ Guardians of all children gave their informed consent prior to inclusion of their children in the study.

\section{Results}

There were 52 children suffering from INS whom we finally included in our study analysis. Mean age of the patients was $4.75 \pm 2.84$ years. Most of the patients were below the age of 8 years (Figure 1).

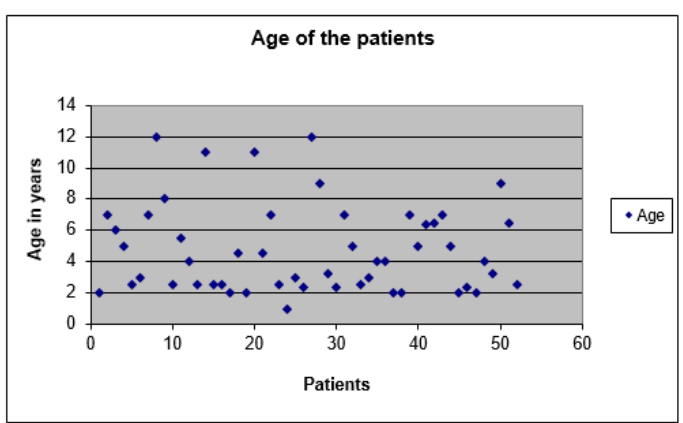

Figure 1: Age distribution of the patients

On subgroup analysis, mean age of patients with FSGS was 4.48 years and MCD was 3.96 years. There were 30 (57\%) males and 22 (43\%) females. Similar sex proportions were noted in subgroups with $62.5 \%$ males among FSGS. A slightly higher male predominance $(71.4 \%)$ was noticed among MCD patients. The numbers of MSGN, MGN andr MPGN patients were too small to comment on age or sex prevalence. Among the 52 patients, 11 (21\%) showed grade 1 stunting according to Waterlow classification and $13(25 \%)$ had evidence of grade 2 stunting (Figure 2).

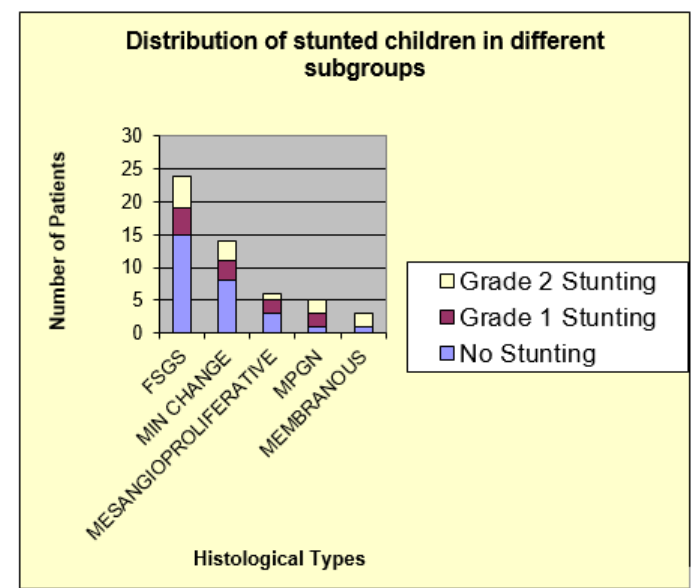

Figure 2: Distribution of stunting in subgroups

Considering weight for height as corroborative of acute malnutrition in children up to 5 years according to WHO Z-score Chart, 5 patients $(15 \%)$ had low weight for height, and 1 patient (3\%) had severe acute malnutrition. Calculating body mass index (BMI) in those above 5 years a higher proportion $37 \%$ (7 out of 19 ) of severe malnutrition was found. 
Most common presentation on admission was body swelling $(100 \%)$. Other clinical presentations were oliguria $(21 \%)$, hypertension $(29 \%)$, haematuria $(52 \%)$, respiratory distress, fever etc. Considering relative frequency of haematuria in different subtypes, macroscopic and microscopic haematuria were present respectively in $16.7 \%$ and $33.3 \%$ of FSGS, $21.4 \%$ and $28.6 \%$ of MCD, $50 \%$ and $16.7 \%$ of MSGN (Figure 3).

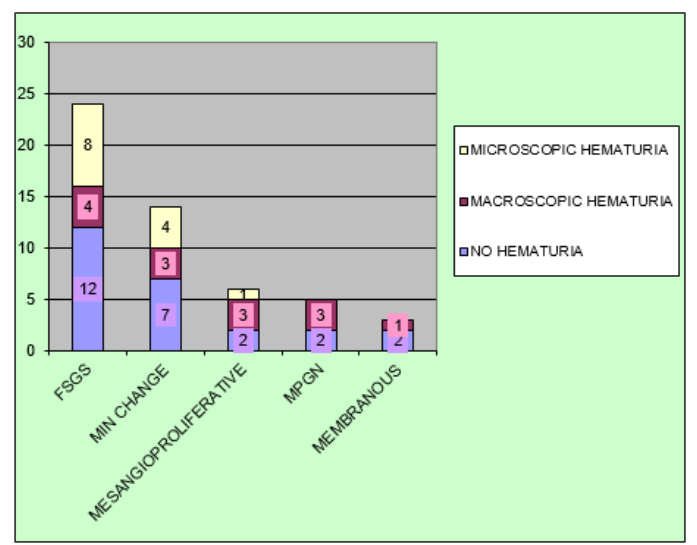

Figure 3: Distribution of haematuria patients between different subgroups

Haematuria was present in $50 \%$ of MCD, compared to $52.6 \%$ in Non-MCD group. The difference was not statistically significant.

In our study 15 (28.8\%) children had hypertension according to BP percentile calculation. Among FSGS patients 2 had Stage 1 hypertension (HTN) and 6 had Stage 2 HTN. (Figure 4).

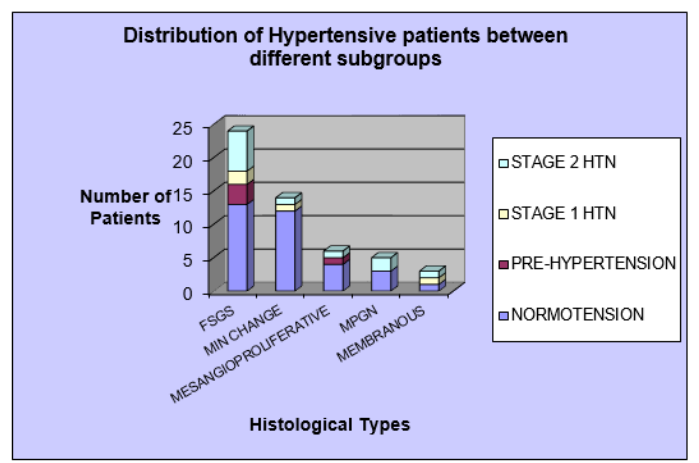

Figure 4: Distribution of hypertensive patients between different subgroups

Comparing the prevalence of hypertension between MCD (14.29\%) and Non-MCD (34.21\%) group, the difference was not significant.
Classifying the patients according to their past history and records of treatment, 11 (21.2\%) patients were suffering from $1^{\text {st }}$ attack of NS, 16 (30.8\%) patients from infrequent relapse and 25 (48.1\%) from frequent relapse (Figure 5).

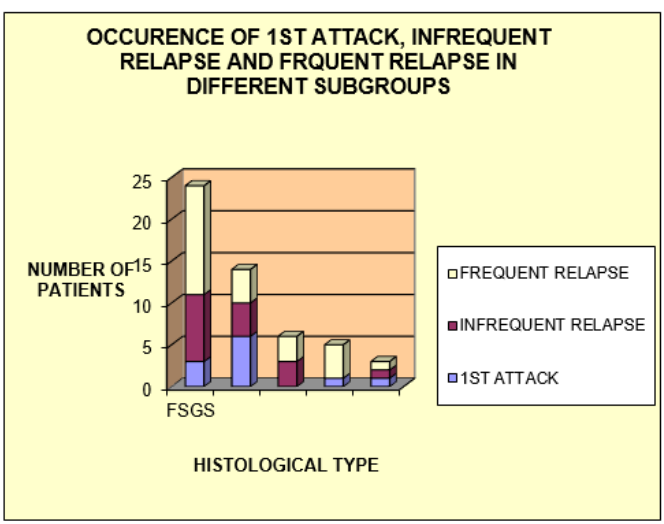

Figure 5: Occurrence of first attack, infrequent relapse and frequent relapse

Comparing the occurrence of relapse between MCD (57.1\%) and Non-MCD (86.8\%) group, the difference was statistically significant $(\mathrm{p}=0.02)$.

Most of the patients suffering from chronic malnutrition were having frequent or infrequent relapses. Among 11 cases of $1^{\text {st }}$ attack, 2 were found to be steroid dependent and 1 patient showed steroid resistance. Oliguria was present in 3 out of $14(21.4 \%)$ patients with MCD and 8 out of 38 (21.1\%) Non-MCD patients (Figure 6)

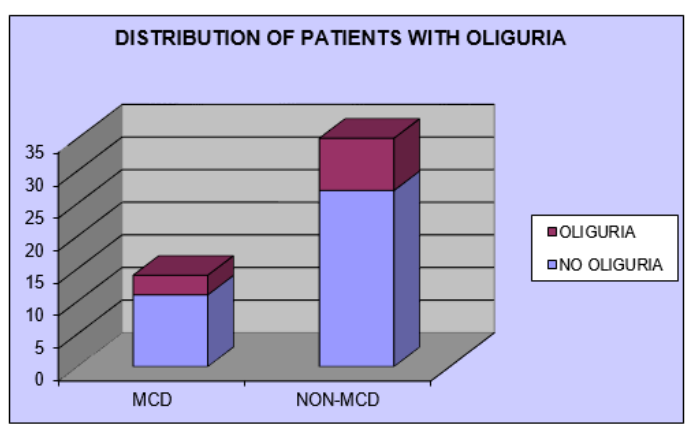

Figure 6: Distribution of patients with oliguria between MCD and non- MCD

The baseline biochemical profile of the patients is shown in Table 1. 
Table 1: Overall baseline mean value \pm standard deviation for different biochemical parameters

\begin{tabular}{|l|c|}
\hline \multicolumn{1}{|c|}{ Biochemical parameter } & Mean value \pm Standard Deviation \\
\hline Haemoglobin $(\mathrm{gm} / \mathrm{dl})$ & $9.99 \pm 2.11$ \\
\hline Total count per cu mm & $9188.46 \pm 2515.96$ \\
\hline Platelet count $(\mathrm{lacs}$ per cu mm) & $5.81 \pm 0.83$ \\
\hline Urea $(\mathrm{mg} / \mathrm{dl})$ & $37.6 \pm 10.24(13.42)$ \\
\hline Creatinine $(\mathrm{ng} / \mathrm{ml})$ & $0.61 \pm 0.21(53.92)$ \\
\hline Sodium $(\mathrm{mEq} / \mathrm{L})$ & $138.9 \pm 5.63$ \\
\hline Potassium $(\mathrm{mEq} / \mathrm{L})$ & $4.42 \pm 0.59$ \\
\hline Cholesterol $(\mathrm{mg} / \mathrm{dl})$ & $(489.65 \pm 127.12)(12.68)$ \\
\hline Triglyceride $(\mathrm{mg} / \mathrm{dl})$ & $420.48 \pm 120.53(4.75)$ \\
\hline High density lipoprotein $(\mathrm{mg} / \mathrm{dl})$ & $41.76 \pm 13.15(1.08)$ \\
\hline Low density lipoprotein $(\mathrm{mg} / \mathrm{dl})$ & $363.79 \pm 136.93(9.42)$ \\
\hline Very low density lipoprotein $(\mathrm{mg} / \mathrm{dl})$ & $84.09 \pm 24.1$ \\
\hline Serum glutamic pyruvate transaminase (IU/L) & $27.29 \pm 11.69$ \\
\hline Serum glutamic oxaloacetic transaminase (IU/L) & $31.6 \pm 13.8$ \\
\hline Alkaline phosphatase $(\mathrm{IU} / \mathrm{L})$ & $231.6 \pm 31.84$ \\
\hline Bilirubin $(\mathrm{mg} / \mathrm{dl})$ & $0.63 \pm 0.29(10.77)$ \\
\hline Albumin $(\mathrm{gm} / \mathrm{dl})$ & $1.8 \pm 0.42(18)$ \\
\hline Globulin $(\mathrm{gm} / \mathrm{dl})$ & $2.13 \pm 0.4$ \\
\hline
\end{tabular}

The baseline biochemical profile of the patients was evaluated. As per WHO recommended age specific cut off for anaemia 36 patients $(69.2 \%)$ were anaemic at presentation. Only 9 patients showed evidence of leucocytosis. Platelet count was on the higher side in most of the patients with a mean of $5.81 \pm 0.83 \mathrm{lacs} / \mathrm{cu} \mathrm{mm}$. Thrombocytosis was observed in $55.8 \%$. Liver function tests revealed normal bilirubin, liver enzymes. Albumin was decreased in all patients in varying degrees with a mean of $1.8 \pm 0.42 \mathrm{~g} / \mathrm{dl}$ at admission (range: 1.1 to $3 \mathrm{~g} / \mathrm{dl}$ ). Globulin was also decreased substantially with a mean of $2.12 \pm 0.4 \mathrm{~g} / \mathrm{dl}$. Renal function tests showed more or less normal limits. If 40 units is taken as cut off for blood urea, 16 patients had a marginally elevated blood urea. Normal values were observed for sodium and potassium. Rise in the lipid fragments of blood, was almost universal within our patients, the most marked and constant feature being hypercholesterolaemia. Mean cholesterol level was $489.65 \pm 127.12 \mathrm{mg} / \mathrm{dl}$. Baseline biochemical reports were compared between MCD and Non-MCD. The differences observed were non-significant (Table 2).

Table 2: Mean value \pm standard deviation and p-value for different biochemical parameters between minimal change disease and non-minimal change disease group

\begin{tabular}{|l|c|c|c|}
\hline \multicolumn{1}{|c|}{ Biochemical parameter } & MCD (n=14) & Non-MCD (n=38) & p-value \\
\hline Haemoglobin (gm/dl) & $9.37 \pm 1.52$ & $10.23 \pm 2.04$ & 0.11 \\
\hline Total count per cu mm & $8642.86 \pm 2099.61$ & $9389.47 \pm 2649.91$ & 0.3 \\
\hline Platelets (lacs per cu mm) & $5.77 \pm .44$ & $5.82 \pm .86$ & 0.89 \\
\hline Urea (mg/dl) & $41.71 \pm 11.17(14.89)$ & $36.08 \pm 9.6(12.88)$ & 0.200681 \\
\hline Creatinine (ng/ml) & $0.53 \pm 0.21(46.85)$ & $0.64 \pm 0.17(56.57)$ & 0.1 \\
\hline Sodium (mEq/L) & $138.07 \pm 5.93$ & $139.24 \pm 5.56$ & 0.53 \\
\hline Potassium (mEq/L) & $4.28 \pm 0.52$ & $4.48 \pm 0.57$ & 0.25 \\
\hline Cholesterol (mg/dl) & $450.64 \pm 102.33(11.67)$ & $504.03 \pm 133.45(13.05)$ & 0.13 \\
\hline Triglyceride (mg/dl) & $411.14 \pm 128.8(4.64)$ & $423.92 \pm 118.95(4.79)$ & 0.75 \\
\hline High density lipoprotein (mg/dl) & $41.93 \pm 9.38(1.09)$ & $41.71 \pm 14.4(1.08)$ & 0.95 \\
\hline Low density lipoprotein (mg/dl) & $326.49 \pm 112.65(8.46)$ & $326.49 \pm 112.65(8.46)$ & 0.19 \\
\hline Very low density lipoprotein (mg/dl) & $82.23 \pm 25.76$ & $84.78 \pm 23.79$ & 0.75 \\
\hline $\begin{array}{l}\text { Serum glutamic pyruvate transaminase } \\
\text { (IU/L) }\end{array}$ & $23.86 \pm 8.89$ & $28.55 \pm 12.4$ & 0.14 \\
\hline $\begin{array}{l}\text { Serum glutamic } \text { oxaloacetic } \\
\text { transaminase (IU/L) }\end{array}$ & $29.93 \pm 6.39$ & & 0.46 \\
\hline Alkaline phosphatase (IU/L) & $231 \pm 38.56$ & $231.82 \pm 29.58$ & 0.94 \\
\hline Bilirubin (mg/dl) & $0.55 \pm 0.32(9.4)$ & $0.65 \pm 0.28(11.11)$ & 0.34 \\
\hline Albumin (gm/dl) & $1.86 \pm 0.3(18.6)$ & $1.78 \pm 0.46(17.8)$ & 0.43 \\
\hline Globulin (gm/dl) & $1.96 \pm 0.41$ & $2.19 \pm 0.37$ & 0.07 \\
\hline
\end{tabular}


Urine examination revealed marked proteinuria in most of the cases. Blood in urine was detected in $23(44.2 \%)$. Culture was positive in $11(21.2 \%)$ patients of which 7 cases were positive for E. coli, 2 cases for Proteus species and 1 case for Klebsiella species

According to histological characteristics of the individual renal biopsy specimens, the most frequently noted glomerular lesion was FSGS in $45 \%$. MCD was noted in $27 \%$, MSGN in $12 \%$, MPGN in $10 \%$ and $\mathrm{MGN}$ in 6\% (Figure 7).

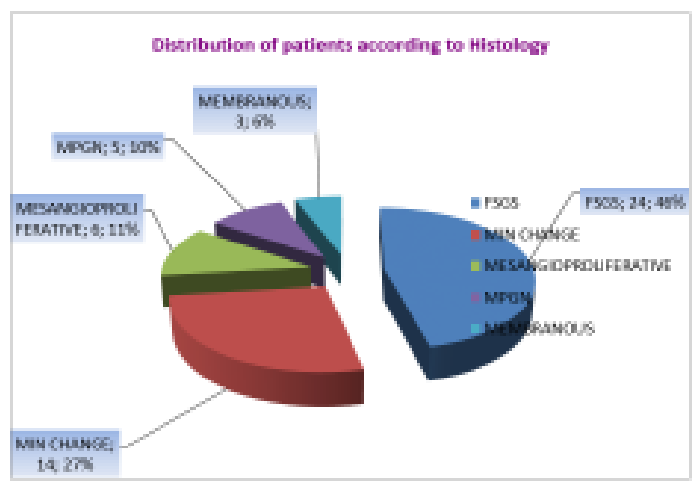

Figure 7: Distribution of patients according to histological lesion in renal biopsy

\section{Discussion}

According to the literature, MCD still remains the most prevalent cause of INS. However our study revealed a different proportion from the usual existing trend with FSGS leading the group in a substantial $45 \%$ of cases, while MCD lagged far behind. A review article by Borges $\mathrm{FF}$ et al. published in Pediatric Nephrology in $2007^{2}$ reviewed the increase in the frequency of FSGS. They compared the frequency of FSGS in two consecutive periods over the past three decades. The results suggested that a shift in the pathological pattern of NS in children might be occurring, resulting in an increase in FSGS frequency. Another large review analysed a 17-year database covering a 275,000-child population with mandatory referral ${ }^{5}$. They confirmed the increasing incidence of FSGS in children with idiopathic NS, but at the same time, found no reason to change the initial therapy and current indications to perform renal biopsy in childhood NS.

A study by Kari JA on determining the changing trends of histopathology in childhood nephrotic syndrome in western Saudi Arabia revealed significant statistical findings ${ }^{6}$. In this study the clinical data of 46 paediatric patients diagnosed with INS between 1997 and 2001, were reviewed and compared with data of 132 patients with INS diagnosed between 1983 and 1992. There was a clear decline in the incidence of MCD in recent years from $79.5 \%$ to $65 \%(\mathrm{p}=0.02)$ (even after assuming that all patients without a histological diagnosis had presumptive MCD and the total incidence of $\mathrm{MCD}=$ biopsy proven + presumptive). The incidence of FSGS was significantly greater in biopsies performed in the recent period, $35 \%$ versus $16.7 \%$ in earlier period $(p=0.05)$. Similarly, they found an increased incidence of MPGN in total patients $13.0 \%$ and group $B, 5.3 \%$, $(p=0.02)$.

Another study conducted in Lucknow, India ${ }^{7}$, involved 600 children with INS. They analysed only those children who underwent biopsies. The study group included 290 children in which adequate biopsy reports were available. FSGS was the most common histopathological subtype, occurring in 38\%. Other subtypes included MCD in $32 \%$, MPGN in $15 \%$, MSGN in $11 \%$, MGN $2 \%$, and diffuse mesangial sclerosis in $1 \%$. However, in children under 8 years of age, MCD was the most common entity, whereas FSGS predominated in children with age at onset greater than 8 years. The age at onset of NS was significantly higher in the non-MCD group than the MCD group. The incidence of hypertension, microhaematuria and gross haematuria was significantly lower in the MCD group. Bonilla-Felix $\mathrm{M}$ et al. ${ }^{8}$ also reported a similar conclusion clarifying the facts that before 1990, FSGS was diagnosed in $23 \%$ of all renal biopsies but increased to $47 \%$ afterward $(\mathrm{P}=0.02)$. This pattern was observed in all ethnic groups. In African Americans, there was a trend for an increase in the incidence of FSGS from $38 \%$ before 1990 to $69 \%$ after 1990. A similar trend was observed in Caucasians (from 20 to 45\%) and Hispanics (from 8 to $33 \%$ ). Hispanics had the highest incidence of MCD (biopsy proven + presumptive: $73 \%$ ), followed by Caucasians (53\%) and African Americans (37\%).

All studies mentioned so far were mostly based on records of patients who underwent renal biopsy according to standard indications. Hence many patients were excluded from having a confirmatory histological diagnosis and were presumed to have MCD. Even among those who underwent biopsy, there was a clear surge noted in the incidence of FSGS. Our study was different in a major aspect, that all patients underwent biopsy (unless there was some contraindication). Our results were varying markedly in view of the fact that FSGS had a clearly higher incidence compared to MCD. So whether FSGS is exceptionally predominant in our set of paediatric population or whether mandatory renal biopsy in all had led to an altered aetiological pattern, remains a matter for debate. 
The mean age of the patients was not significantly different between MCD and non-MCD, being 4.2 vs 5.08 years respectively. Most of our patients were below the age of 8 years (Figure 1). The median age at presentation was similarly 4.5 years in the study by Ahmadzadeh Ali et al. ${ }^{19}$. According to Nelson Textbook of Paediatrics, 18th ed. the male: female ratio among nephrotic patients is approximately 2:1. International Study of Kidney Disease in Children (ISKDC) data indicate that $66 \%$ of patients with either MCNS or FSGS are male, whereas $65 \%$ of individuals with MPGN are female. Our findings were corroborative in this aspect with male: female ratio of around 5:2 in MCD, 5:3 in FSGS and 2:1 MSGN. All five patients with MPGN were females and all three patients with MGN were males. Overall there were $58 \%$ males and $42 \%$ females. Comparing the occurrence of relapse (frequent and infrequent) between MCD and Non-MCD group, the difference was statistically significant $(p=0.02)$.

Haematuria is distinctly unusual in INS. The frequency of macrohaematuria depends on the histological subtype being more common in those patients with MPGN than in other causes, but its frequency in MCNS has been reported to be as high as $3-4 \%$ of cases. Statistically, a higher percentage of patients with FSGS have microhaematuria than those with MCNS. Among our patients, macroscopic and microscopic haematuria were present respectively in $16.7 \%$ and $33.3 \%$ of FSGS, $21.4 \%$ and $28.6 \%$ of MCD. Macroscopic haematuria was present in $60 \%$ of MPGN and $33.3 \%$ of MGN (Figure 3). Haematuria was present in $50 \%$ of $\mathrm{MCD}$, compared to $52.6 \%$ in Non-MCD group, the difference being statistically nonsignificant. A study conducted in Iran revealed $34 \%$ children had haematuria. All patients with gross haematuria underwent renal biopsy indicating FSGS in $60 \%$ and MSGN and MPGN in $20 \%$ each ${ }^{9}$.

Hypertension is more common in children with FSGS and MPGN rather than MCNS. In the ISKDC series, diastolic hypertension was found in $13 \%$ of patients ${ }^{10}$. Nelson Textbook of Paediatrics, 18 th ed. documented a prevalence of hypertension in $35 \%$ of MPGN and $20 \%$ of FSGS in comparison to only $10 \%$ of MCD. In our study, 15 (28.8\%) children had hypertension. Among FSGS, MGN, MSGN and MPGN patients there was 30-40\% prevalence of hypertension (Figure 4). Comparing the prevalence of hypertension among MCD (2 out of 14: $14.3 \%$ ) and Non-MCD (13 out of $38 ; 34.2 \%$ ) group, the difference was not significant. Oliguria may sometimes be associated with Non-MCD as well as MCD patients. Even acute oliguric renal failure as the presenting symptom of MCD has been reported ${ }^{10}$. Oliguria was present in 3 out of 14
(21.4\%) patients with $\mathrm{MCD}$ and 8 out of 38 (21.1\%) non-MCD patients (Figure 6). The difference was not significant.

Preceding respiratory tract infection was noted in 4 $(7.7 \%)$ patients, of which $2(3.9 \%)$ cases were associated with relapse. Preceding urinary tract infection was noted in $7(13.5 \%)$ patients, of which $5(9.6 \%)$ cases were associated with relapse. Upper respiratory infections, otitis media and other infections are often associated with relapses of INS as well. A hypersensitivity event, such as a reaction to bee sting or poison ivy, has been reported to precede the onset of $\mathrm{INS}^{11}$. Evidence of peritonitis was noted in $6(11.5 \%)$ of our patients. Peritonitis caused by either gram-negative or gram-positive organisms remains a serious complication of the $\mathrm{NS}^{12,13,14}$. Children of African American descent appear to be at greater risk for peritonitis ${ }^{15,16}$.

Complete haemogram revealed that 36 patients $(69.2 \%)$ were anaemic at presentation. Relative iron deficiency with lower serum iron and ferritin levels, lower levels of erythropoietin and decreased physiological response to anaemia may be attributed to this anaemia ${ }^{17}$. Chronic malnutrition may also have played its part. Platelet count was on the higher side in most of the patients with a mean of $5.81 \pm .83 \mathrm{lacs} / \mathrm{cu} \mathrm{mm}$. Thrombocytosis was observed in $55.8 \%(\mathrm{n}=29)$ patients taking 4.5 lacs/cu $\mathrm{mm}$ as the upper cut off. The high risk of thromboembolic complications in patients with a nephrotic syndrome have been ascribed to various abnormalities in plasmatic coagulation including increased production of fibrinogen, Factor $\mathrm{V}$ and VIII, reduced fibrinolysis, etc. The following platelet abnormalities were also seen in various studies: increased platelet counts, enhanced platelet adhesiveness, increased spontaneous aggregation and increased heparin neutralizing activitv (PF 4 funct. $)^{18}$. Our incidental finding of thrombocytosis further strengthens these aspects of platelet abnormalities. As platelet aggregation and other relevant studies in this regard were not included in our protocol we did not perform any further investigations in this line.

Liver function tests revealed normal bilirubin \& liver enzymes. Albumin was decreased in all patients in varying degrees with a mean of $1.8 \pm$ $0.42 \mathrm{~g} / \mathrm{dl}$ at admission (range: 1.1 to $3 \mathrm{~g} / \mathrm{dl}$ ). Globulin was also decreased substantially with a mean of $2.12 \pm 0.4 \mathrm{~g} / \mathrm{dl}$. Renal function tests showed more or less normal limits. Blood urea was marginally elevated in 14 patients. These biochemical parameters were compared between MCD and Non-MCD (Table 2). Rise in the lipid fragments of blood, was almost universal within our patients, the most marked and constant feature being hypercholesterolaemia. Among our patients, 
2 children (4.9\%) had positive Mantoux test considering $10 \mathrm{~mm}$ as cut off for immunocompetent children. These two patients were suffering from $1^{\text {st }}$ attack and had to be treated by anti-tuberculous drugs for 2 weeks before starting steroid therapy.

\section{Conclusions}

A mandatory renal biopsy in all patients with INS revealed an enhanced prevalence of FSGS and also suggested that MCD now lags behind FSGS in terms of causation.

\section{References}

1. Eddy AA, Symons JM. Nephrotic syndrome in childhood. Lancet 2003; 362: 629-39.

http://dx.doi.org/10.1016/S01406736(03)1

4184-0

1. Borges FF, Shiraichi L, da Silva MP, Nishimoto EI, Nogueira PC. Is focal segmental glomerulosclerosis increasing in patients with nephrotic syndrome? Pediatric Nephrology 2007; 22(9):1309. 13.

http://dx.doi.org/10.1007/s00467-007-

0516-0

PMid: 17551756

2. Boyer O, Moulder JK, Somers MJ. Focal and segmental glomerulosclerosis in children: a longitudinal assessment. Pediatric Nephrology 2007; 22(8):115966.

http://dx.doi.org/10.1007/s00467-007-

0493-3

PMid: 17437129

3. Bagga A, Srivastava RN. Nephrotic syndrome. In: Srivastava RN, Bagga A, editors. Pediatric Nephrology. 4th ed. New Delhi: Jaypee; 2005 p. 159-200.

4. Filler G, Young E, Geier P, Carpenter B, Drukker A, Feber J. Is there really an increase in non-minimal change nephrotic syndrome in children? American Journal of Kidney Disease 2003; 42(6):1107-13. http://dx.doi.org/10.1053/j.ajkd.2003.08.0

10

PMid: 14655180
5. Kari JA. Changing trends of histopathology in childhood nephrotic syndrome in western Saudi Arabia. Saudi Medical Journal 2002; 23(3):317-21.

PMid: 11938425

6. Kumar J, Gulati S, Sharma AP, Sharma RK, Gupta RK. Histopathological spectrum of childhood nephrotic syndrome in Indian children. Pediatric Nephrology 2003; 18(7):657-60.

PMid: 12743793

7. Bonilla-Felix M, Parra C, Dajani T, Ferris M, Swinford RD, Portman RJ, Verani R. Changing patterns in the histopathology of idiopathic nephrotic syndrome in children. Kidney Int. 1999; 55(5):1885-90.

http://dx.doi.org/10.1046/j.15231755.1999 .00408.x

PMid: 10231451

8. Ahmadzadeh ali, derakhshan ali, hakimzadeh Mehran, zolfigol ali. Idiopathic nephrotic syndrome in Iranian children. Indian Pediatrics 2008; 45(17):

9. The primary nephrotic syndrome in children. Identification of patients with minimal change nephrotic syndrome from initial response to prednisone. A report of the International Study of Kidney Disease in Children. Journal of Pediatrics 1981; 98:561-4.

http://dx.doi.org/10.1046/j.15231755.1999 .00408.x

PMid: 10231451

10. Niaudet P. Steroid-Sensitive Idiopathic Nephrotic Syndrome in Children. In: Avner E, Harmon W Niaudet P. Pediatric Nephrology. $5^{\text {th }}$ ed. 2004: chap 27.

11. Hulter HN, Bonner EL Lipoid nephrosis appearing as acute oliguric renal failure. Jr Arch Intern Med. 1980; 140(3):403-5. http://dx.doi.org/10.1001/archinte.140.3.4 03

12. Gorensek MJ, Lebel MH, Nelson JD. Peritonitis in children with nephrotic syndrome. Pediatrics 1988; 81: 849-56. PMid: 3368284 
13. Feinstein EI, Chesney RW, Zelikovic I: Peritonitis in childhood renal disease. American Journal of Nephrology 1988; 8:147-65. http://dx.doi.org/10.1159/000167575

14. Krensky AM, Ingelfinger JR, Grupe WE. Peritonitis in childhood nephrotic syndrome: 1970-1980. American Journal of Disease in Children 1982; 136:732-6. http://dx.doi.org/10.1001/archpedi.1982.0 3970440076023

15. Rubin HM, Blau EB, Michaels RH: Haemophilus and pneumococcal peritonitis in children with the nephrotic syndrome. Pediatrics 1975; 56:598-601. PMid: 241048
16. Recommendation of the Immunization Practices Advisory Committee. Pneumococcal polysaccharide vaccine. Annals of Internal Medicine 1982; 96:2035. http://dx.doi.org/10.7326/0003-4819-96-2203

PMid: 6277218

17. Feinstein S, Becker-Cohen R, Algur N, Raveh D, Shalev H, Shvil Y, Frishberg Y. Erythropoietin deficiency causes anaemia in nephrotic children with normal kidney function. American Journal of Kidney Disease 2001; 37: 736-42. http://dx.doi.org/10.1016/S02726386(01)8 $\underline{0122-0}$ 